\title{
Acciones para el desarrollo de la inteligencia emocional en los cuadros del Hotel NH Capri
}

DOI: https://doi.org/10.33262/ap.v2i2.30

\begin{abstract}
(c) (i) (우)
Actions for the development of emotional intelligence in the pictures of the NH Capri Hotel
\end{abstract}

Rafael Enrique Calzado Morales., ${ }^{1}$ Yulima Valdés Bencomo. ${ }^{2}$ \& Yasser Vázquez Alfonso. ${ }^{3}$

\begin{abstract}
.
The purpose of this research is to propose actions for the development of emotional intelligence in the NH Capri hotel executives. The research that was developed is a nonexperimental descriptive type of cross-section. For the development of the present study, an evaluation of scientific observation with indicators in each of the dimensions of emotional intelligence was started, since it allowed a deeper approach to the object of study. This research was used during the training process to analyze the current behavior of managers. Other data collection instruments were applied such as: Diagnostic Questionnaire and Interview. These instruments were applied to a sample of 8 managers. The sample was selected based on an intentional non-probabilistic sampling that took into account selection criteria. The results obtained show the evaluation of managers in each of the dimensions of emotional intelligence. As a general result it is evident that, the managers evaluated have a medium-low level of emotional intelligence, so it is necessary to develop and implement said ability to achieve good performance in the workplace. The proposal of actions to develop emotional intelligence ensures that managers expand their ability to influence, better manage
\end{abstract}

\footnotetext{
${ }^{1}$ Facultad de Turismo, Universidad de la Habana, Cuba. yalfos1@gmail.com

${ }^{2}$ Facultad de Turismo, Universidad de la Habana, Cuba. ybencomo@ftur.uh.cu

${ }^{3}$ Facultad de Turismo, Universidad de la Habana, Cuba. yalfos1@gmail.com
} 
interpersonal relationships, promote their psychological well-being and improve their work performance, increasingly motivating themselves to efficiently and effectively perform their role as manager.

Keywords: ability, management skills, intelligence, emotion, emotional intelligence.

\section{Resumen.}

La presente investigación tiene como objetivo general proponer acciones para el desarrollo de la inteligencia emocional en los cuadros del hotel NH Capri. La investigación que se desarrolló es de tipo descriptivo no experimental de corte transversal. Para el desarrollo del presente estudio se partió de una evaluación de la observación científica con indicadores en cada una de las dimensiones de la inteligencia emocional, ya que posibilitó un acercamiento más profundo al objeto de estudio. Se aplicaron otros instrumentos de recolección de datos como: Cuestionario Diagnóstico y Entrevista. Estos instrumentos fueron aplicados a una muestra de 8 directivos. La selección de la muestra se realizó a partir de un muestreo no probabilístico intencional que tuvo en cuenta criterios de selección. Los resultados obtenidos muestran la evaluación de los directivos en cada una de las dimensiones de la inteligencia emocional. Como resultado general se evidencia que, los directivos evaluados presentan un nivel medio-bajo de inteligencia emocional, por lo que se hace necesario el desarrollo e implementación de dicha habilidad para poder alcanzar buen desempeño en el puesto de trabajo. La propuesta de acciones para desarrollar la inteligencia emocional garantiza que los directivos amplíen su capacidad de influencia, manejen mejor las relaciones interpersonales, favorezcan su bienestar psicológico y mejoren su rendimiento laboral motivándose cada vez más en desempeñar eficiente y eficazmente su rol como directivo.

Palabras claves: habilidad, habilidades directivas, inteligencia, emoción, inteligencia emocional.

\section{Introducción.}

El desarrollo de la tecnología, la información, el conocimiento, los impactos y retos que enfrentan las empresas han generado la necesidad de la creación e implementación de un enfoque de dirección flexible, innovador, proactivo y motivador, capaz de comprometer el talento humano para el logro eficiente y eficaz de la misión de las organizaciones, surgiendo así el enfoque estratégico en la dirección como vía de solución eficiente a esos retos (Ronda, 2007).

La competitividad de una empresa se sustenta en las competencias y habilidades que posean sus directivos, lo que se demuestra cuando se comprueba su funcionamiento. En este sentido, la efectividad organizacional resultante se traduce en el nuevo valor que se genera, a partir 
del proceso de capacitación, con el objetivo de cumplir las expectativas de la sociedad (Martínez, 2017).

En la actualidad a nivel internacional la temática de las habilidades directivas en el ejercicio de la dirección estratégica de entidades turísticas constituye una preocupación de primer orden, producto a las nuevas tendencias de investigación en este campo que han demostrado que el esplendor en el desarrollo organizacional se centraliza en las habilidades de los directores para la toma de decisiones estratégicas, en función de los objetivos organizacionales como categoría rectora de sus procesos.

La inteligencia emocional resulta ser una habilidad que ocupa cada vez un lugar más destacado y necesario en las personas, para hacer frente a las múltiples incidencias que se presentan en el desempeño laboral del día a día, para ello, siguiendo a Goleman (1998), se vive en una época en la que la perspectiva de futuro depende de la capacidad de controlarse a uno mismo y de manejar adecuadamente nuestras relaciones.

El conocimiento de la inteligencia emocional de un directivo es importante, ya que de él depende el trabajo de otras personas, que se ven influenciadas por el despliegue de la misma, afectando esto al conjunto de la organización. Las organizaciones afrontarán mejor las exigencias de un entorno tan exigente y cambiante si están integradas por directivos y trabajadores con inteligencia emocional, capaces de hacer frente a cambios y desempeñar sus responsabilidades con mayor eficacia. Esos directivos valoran el trabajo en equipo, la cooperación con otras personas, la capacidad de adaptación y la orientación al logro (Calzado; 2020).

El directivo debe ser capaz de manejar situaciones complejas para optimizar sus resultados a partir del conocimiento profundo de la naturaleza humana, sus expectativas, necesidades y motivaciones, para lo cual es necesario, capacidad para comprender (empatía), saber generar confianza entre las personas que dirige (manejo de relaciones), saber escuchar, comunicar de forma convincente, generando ilusión, entusiasmo y compromiso en las personas, a través de objetivos y proyectos ilusionantes.

En el contexto actual de Cuba y específicamente en el sector turístico, se hace necesario un desarrollo en las empresas con la finalidad de lograr un mejoramiento en los sistemas de dirección y en la gestión integral de las mismas para alcanzar resultados eficientes. Dando seguimiento a lo establecido por la política del Perfeccionamiento Empresarial en los decretos 252 sobre la continuidad y el fortalecimiento del Sistema de Dirección y Gestión Empresarial cubano y el Decreto Ley 281 sobre las principales normas de actuación y procedimientos técnicos del Sistema de Dirección y Gestión Empresarial Estatal. De este modo, la capacitación de los directivos cubanos en la habilidad de inteligencia emocional, para el perfeccionamiento de sus funciones en el proceso administrativo, es un requisito para su efectivo desenvolvimiento en su puesto de trabajo. 
Muchas son las instalaciones turísticas cubanas donde se puede apreciar la ausencia de un desarrollo de la habilidad inteligencia emocional por los mandos a diferentes niveles. Un estudio realizado en el hotel NH Capri perteneciente a la cadena hotelera Gran Caribe, arrojó que, en la actualidad, sus directivos muestran un bajo nivel de inteligencia emocional; situación a la cual, de no prestarles la debida atención, puede repercutir negativamente en los resultados del hotel. Todo esto hace necesario, plantear nuevas fórmulas para la mejora de esta habilidad.

Ante esta situación problémica se plantea como objetivo general de la presente investigación: Proponer acciones para el desarrollo de la inteligencia emocional en los cuadros del hotel NH Capri.

\section{Materiales y métodos.}

La metodología aplicada es cualitativa, pues se incluye una variedad de concepciones, visiones, técnicas y estudios no cuantitativos. Además, se exploran los fenómenos en profundidad, se conduce básicamente en ambientes naturales, los significados se extraen de los datos y no se fundamenta en la estadística. Las investigaciones no experimentales son estudios que se realizan sin la manipulación deliberada de variables y en los que sólo se observan los fenómenos en su ambiente natural para después analizarlos. Además, plantea que dentro de esta existe el enfoque transaccional o transversal, el cual recolecta datos en un solo momento, en un tiempo único. Entre sus tipos, los estudios descriptivos buscan especificar propiedades, características y los perfiles de personas, grupos, comunidades, procesos, objetos o cualquier otro fenómeno que se someta a un análisis (Hernández, 2010). Por consiguiente, la investigación que se desarrolló es de tipo descriptivo no experimental de corte transversal, por tratarse de la búsqueda de propiedades y características que se realizan sin la manipulación deliberada de variables y en la que sólo se observan los fenómenos en su ambiente natural, en un momento dado para después ser analizados.

Para la realización de la presente investigación se emplearon los siguientes métodos científicos:

\section{De orden teórico:}

Histórico-Lógico: El método histórico permitió el análisis de la evolución teórica de la entidad desde su fundación hasta la fecha para el estudio de las habilidades directivas y la inteligencia emocional como habilidad esencial.

Hipotético-deductivo: Su empleo facilitó la formulación de la hipótesis de la investigación como relación entre las variables habilidades directivas e inteligencia emocional y las acciones para su desarrollo.

Inducción-Deducción: Deducción es la conclusión que se formula sobre el caso particular partiendo de lo general, mientras la inducción parte de la observación de los hechos o 
fenómenos particulares para obtener una conclusión general. Es empleado durante toda la investigación desde los elementos teóricos encontrados en la literatura relacionados a la inteligencia emocional, al Capri, y para el análisis de los resultados e interpretación general de la situación para arribar a conclusiones.

Análisis-síntesis: Posibilitó la búsqueda y el procesamiento de la información a partir de la bibliografía consultada y del estudio teórico de la temática investigada. Facilitará determinar las características del estudio de la inteligencia emocional de los cuadros vinculadas a la gestión de alojamiento.

\section{De orden empírico:}

Observación Científica: Es un método que posibilita un acercamiento más profundo al objeto de estudio, complementando la información obtenida por medio de otros métodos y técnicas. En esta investigación se utilizó durante el proceso de entrenamiento para analizar el comportamiento de los directivos, a través de una guía de observación en formato de criterios valorativos.

Cuestionario Diagnóstico: se realizó con el objetivo de recopilar información real acerca del nivel de inteligencia emocional que presentan los directivos.

Clave de resultados: las afirmaciones se reordenaron de acuerdo con las dimensiones de la inteligencia emocional evaluadas. Los números que aparecen junto a cada alternativa indican la cantidad de expertos que seleccionaron esa alternativa.

Entrevista: Fue realizada a la directora general tuvo por objetivo interpretar la información, impresiones, intenciones y pensamientos del entrevistado hacia la inteligencia emocional como habilidad directiva. En este estudio se entendió la entrevista como encuentros entre el entrevistador y el entrevistado, el cual estuvo dirigido a la comprensión de las perspectivas que tiene el entrevistado de acuerdo a su vida, experiencia o situación dentro de la instalación, tal como lo expresan sus propias palabras. Se respetó por encima de cualquier consideración lo dicho por el entrevistado.

Búsqueda referencial en Internet: Una buena parte de la literatura utilizada se obtuvo de fuentes digitales, ofreciendo así Internet una gran cantidad de información importante, dada la actualidad y el desarrollo de teorías sobre los temas a investigar.

La población de estudio comprende a los directivos del hotel NH Capri, pertenecientes a la cadena hotelera Gran Caribe. La muestra total para realizar la propuesta de acciones fue de 8 trabajadores de cargos directivos. La selección de la muestra se realizó a partir de un muestreo no probabilístico intencional que tuvo en cuenta los siguientes criterios de selección:

- Los directivos deben ser trabajadores del hotel objeto de estudio. 
- Deben estar dispuestos a colaborar con la investigación.

- Deben tener no menos de 3 años de experiencia en cargos de dirección.

La investigación se realiza en el Hotel NH Capri, está situado en la intersección de las calles $\mathrm{N}$ y 21 en la céntrica zona del Vedado y a pocos metros del malecón Habanero. Ha sido renovado completamente acorde a las tendencias contemporáneas sin apartarse de su concepción original. Conserva su encanto de los años 50, es acogedor, bien confortable y funcional. Ideal para hombres de negocios, grupos de incentivos, estancias de ocio y lunas de miel. Su arquitectura consta de un total de 19 pisos y 220 habitaciones. Es un hotel 4 estrellas que ofrece un servicio de alta calidad, posee estándares y variedad en su oferta al nivel de un hotel 5 estrellas. Es administrado por la cadena hotelera Gran Caribe y por la prestigiosa cadena Española NH Hotels Group.

El constructo Inteligencia Emocional (IE) tiene sus orígenes en los estudios de Edward (1920) sobre la inteligencia Social, definida como la capacidad para comprender y dirigir a las personas y gestionar sus relaciones. Más tarde, Gardner (1983) abundó en los conceptos de inteligencia interpersonal e intrapersonal señalando que la primera, la inteligencia interpersonal, denota la capacidad para comprender las intenciones, motivaciones y deseos de los demás para trabajar eficazmente con ellos y, la segunda, la inteligencia intrapersonal, como la capacidad para comprenderse a uno mismo de forma efectiva.

En cambio, no fue hasta Mayer (1990) cuando se realizaron las primeras investigaciones relevantes sobre este concepto. Estos autores se enfocaron en componentes afectivos, emocionales, personales y sociales (sin menospreciar los cognitivos) que suponían un factor clave de éxito en los diferentes ámbitos de la vida. Sin embargo, fue Goleman (1995) quien realmente popularizó la inteligencia emocional identificando los atributos necesarios para triunfar en la vida y ser feliz, además, explicó como la inteligencia emocional no es un factor inamovible, sin capacidad de modificación, sino que es un constructo que es posible desarrollar y potenciar a lo largo de la vida.

Goleman (1998) define la inteligencia emocional como: "La capacidad de motivarnos a nosotros mismos, de perseverar en el empeño a pesar de las posibles frustraciones, de controlar los impulsos, de diferir las gratificaciones, de regular nuestros propios estados de ánimo, de evitar que la angustia interfiera con nuestras facultades racionales". Dicha definición es la que se toma como referencia teórica en esta investigación.

Cooper (1998), refiere la inteligencia emocional como aquello que nos motiva a buscar nuestro potencial único y nuestro propósito que activa nuestros valores íntimos y aspiraciones, transformándolos en cosas en las cuales pensamos. Es también la capacidad de sentir, entender y aplicar eficazmente el poder y la agudeza de las emociones como fuente de energía humana, información, conexión e influencia. 
Según Bello (2012), este concepto constituye una perspectiva optimista del ser humano y explica por qué lo que se consideraba inteligencia en cierto momento, ya no es suficiente para conocer cómo alcanzar el éxito en la actualidad, pues en él queda implícito que cuando la persona tiene un conocimiento eficaz sobre su inteligencia emocional puede encauzar, dirigir y aplicar sus emociones, permitiendo así que las mismas trabajen a su favor, y no en su contra, guiando, por tanto, todas las actitudes de nuestra vida hacia pensamientos constructivos, que mejoren en forma absoluta los resultados finales que queremos alcanzar.

\section{Las dimensiones de la Inteligencia Emocional.}

Tomando como base la propuesta de Goleman, los aportes de Shapiro y otras investigaciones sobre capacidades emocionales, a continuación, se realiza una breve referencia al comportamiento de las dimensiones emocionales.

El autoconocimiento: Se refiere a la capacidad para reconocer y comprender los sentimientos, emociones y necesidades propias, así como sus causas y efectos, en un momento determinado. Implica conocer las propias fortalezas y debilidades a partir de una autovaloración realista y de tener confianza en uno mismo. Esta dimensión constituye según Goleman "la piedra angular de la inteligencia emocional", porque sobre la misma se desarrollan las demás, sobre todo el autocontrol y la empatía; además resulta importante para la introversión psicológica, como guía en la toma de decisiones y para facilitar la comunicación.

El autocontrol: Se refiere a la capacidad para manejar adecuadamente las emociones perturbadoras y las que pueden ser conflictivas e intervenir negativamente en las actividades que realizamos. Es la capacidad para controlar los estados e impulsos, de reorientar las emociones de modo que nos permita afrontar cambios, situaciones de tensión y lograr un equilibrio emocional, no mediante la supresión de la emoción, sino mediante su control adecuándola a las circunstancias.

La automotivación: Se refiere a la capacidad de ordenar las emociones al servicio de un objetivo. Es motivarse por el logro de una meta, tener claridad y valorar la misma como posible, desear el éxito y ser capaz de luchar por él, aprovechar las oportunidades y superar los contratiempos con creatividad, perseverancia y optimismo. Para automotivarse es necesario el control de la vida emocional y la subordinación de la misma para alcanzar un logro.

La empatía: Constituye la capacidad para comprender los sentimientos, emociones, preocupaciones, necesidades y puntos de vista de los otros, lo que permite una adecuada interacción con los otros, y un manejo factible de las problemáticas y emociones ajenas, además de un aprovechamiento y adaptación a la diversidad existente entre las personas. Dado lo anterior, suele decirse que la empatía es la base de las relaciones sociales. Según Goleman (1996) La empatía se asienta en la conciencia emocional de uno mismo, pues cuanto 
más abiertos se está a las propias emociones más capacidad se tendrá para interpretar los sentimientos del otro.

El manejo de relaciones: Se refiere al manejo adecuado de las emociones en las relaciones interpersonales. Consiste en la capacidad para persuadir, influir, inspirar, dirigir, comunicar y negociar con los demás, ya sea una persona o un grupo. Es la capacidad que subyace a la popularidad, la facilidad para formar equipos y colaborar con los otros en el logro de una meta común. Esta dimensión resulta ser la más inclusiva; tiene en su base el resto de las capacidades emocionales en tanto exige la efectividad del manejo de la propia vida afectiva para el manejo de las relaciones con los demás.

\section{Resultados y discusión.}

En la figura 1 se muestra de forma detallada los resultados de la observación en cada uno de las dimensiones de la inteligencia emocional. En la dimensión autoconocimiento se aprecia a partir de las evaluaciones que el nivel de los directivos es medio-bajo. En la dimensión autocontrol se estima a partir de las evaluaciones que el nivel de los directivos es medio. En la dimensión automotivación se valora que el nivel de los directivos es medio. En la dimensión empatía las evaluaciones demuestran que el nivel de los directivos es medio. En la dimensión manejo de relaciones se evidencia de acuerdo con las evaluaciones que el nivel de los directivos es medio-bajo. En conclusión, la observación arroja como resultado final que los directivos del hotel objeto de estudio tienen un nivel de inteligencia emocional mediobajo.

Figura 1. Evaluación de la observación de acuerdo a las dimensiones.

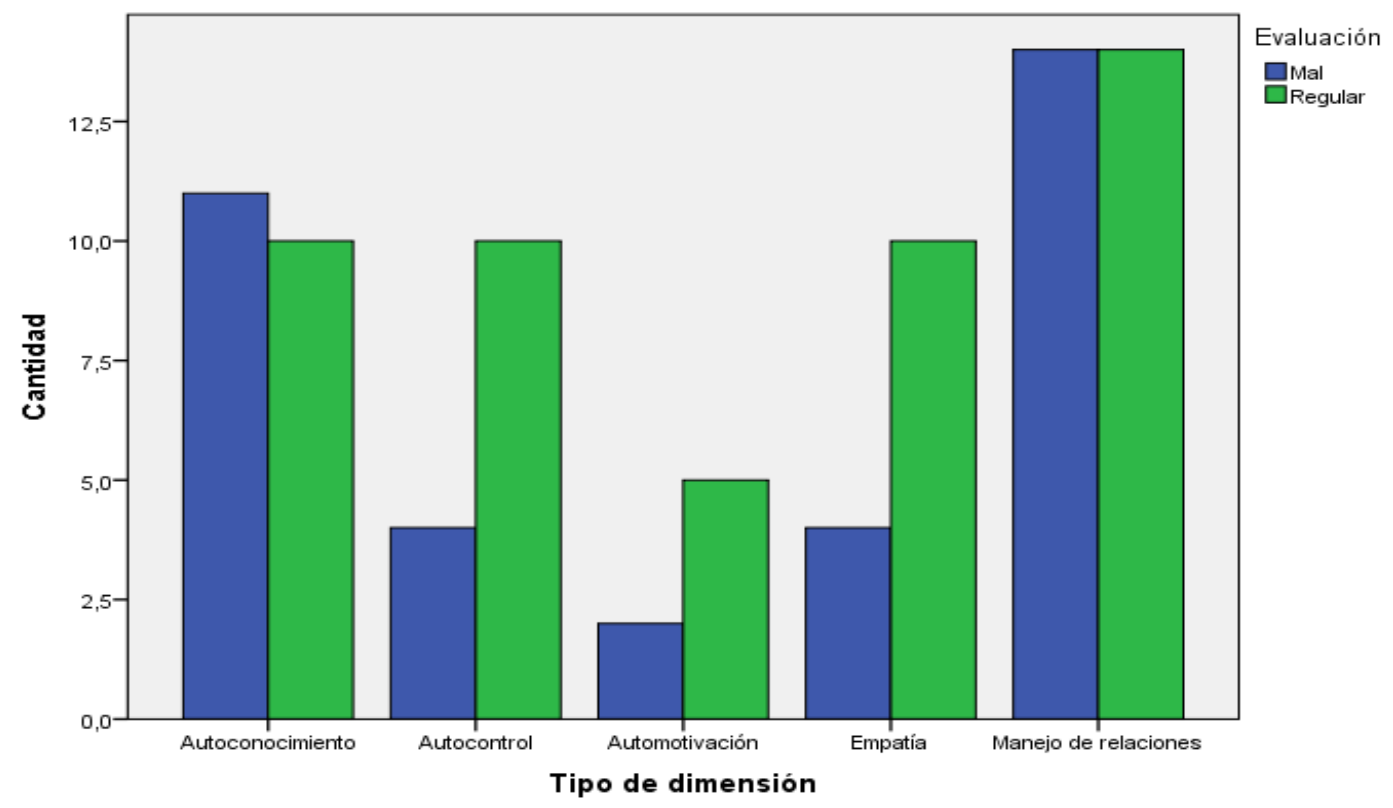

Fuente: Elaboración propia. 
El análisis de la conciencia emocional (Autoconocimiento) se muestra en la figura 2, donde el reactivo 1 la alternativa (a) indica que se está consciente de lo que ocurre emocionalmente en su interior, la alternativa (b) indica que tiene poca consciencia emocional, y la alternativa (c) indica que no se tiene conciencia emocional. Se puede notar que la mayoría de los expertos eligieron la alternativa (c) como respuesta, lo que refleja que no reconocen sus emociones. En el reactivo 5 la alternativa (a) podría estar bien si está consciente de sus prioridades, la alternativa (b) indica que está consciente de posibles puntos de vista alternativos y la alterativa (c) no es adecuada tratándose de un directivo. En este reactivo las alternativas con mayor cantidad de respuestas fueron la (a) y la (c), lo que demuestra que los expertos no tienen conciencia de sus prioridades.

En el reactivo 7 sólo la alternativa (c) indica que está consciente de sus propias reacciones emocionales, sin embargo, la alternativa (a) demuestra el compromiso que tiene con el trabajo. Se define que la mayoría de los expertos están comprometidos con su trabajo, pero, no tienen consciencia de sus propias reacciones emocionales.

En síntesis, se aprecia que los reactivos 1, 5 y 7 responden a la dimensión autoconocimiento y expresan que los expertos no tienen la capacidad para reconocer y comprender los sentimientos, emociones y necesidades propias, así como sus causas y efectos, en un momento determinado. Esto implica que no conocen sus propias fortalezas y debilidades.

Figura 2. Comportamiento de la conciencia emocional (Autoconocimiento).

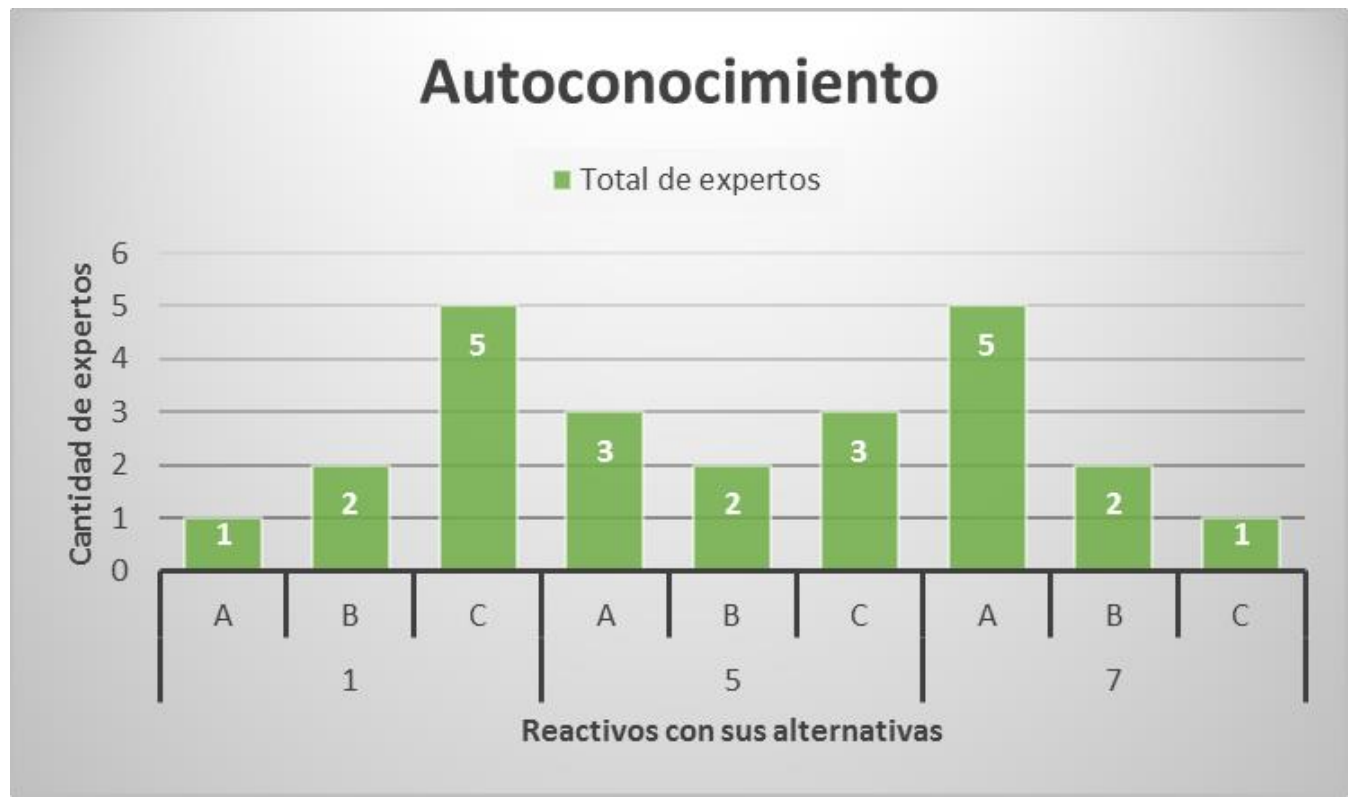

Fuente: Elaboración propia. 
Con respecto al estudio sobre el control emocional (equilibrio) que visualiza la figura 3, se observa que el reactivo 2 la alternativa (a) significa que no confronta el sistema y no expresa sus emociones, la alternativa (b) confronta el sistema, pero no en presencia de los afectados y la alternativa (c) implica que se tiene la confianza suficiente para manejar la situación que lo ocupa. Se evidencia que la mayoría de los expertos adoptaron la alternativa (b) como respuesta, lo que expresa que son capaces, aunque con algunas dificultades de controlar las emociones. En el reactivo 8 sólo la alternativa (c) demuestra control emocional. La alternativa (a) expresa el descontrol emocional. Se percibe que la mayoría de los expertos son capaces de controlar sus emociones.

En conclusión, los reactivos 2 y 8 pertenecientes a la dimensión autocontrol demuestran que los expertos tienen la capacidad, aunque con algunas dificultades para manejar adecuadamente las emociones perturbadoras y las que pueden ser conflictivas e intervenir negativamente en las actividades que realizan.

Figura 3. Comportamiento del control emocional (equilibrio).

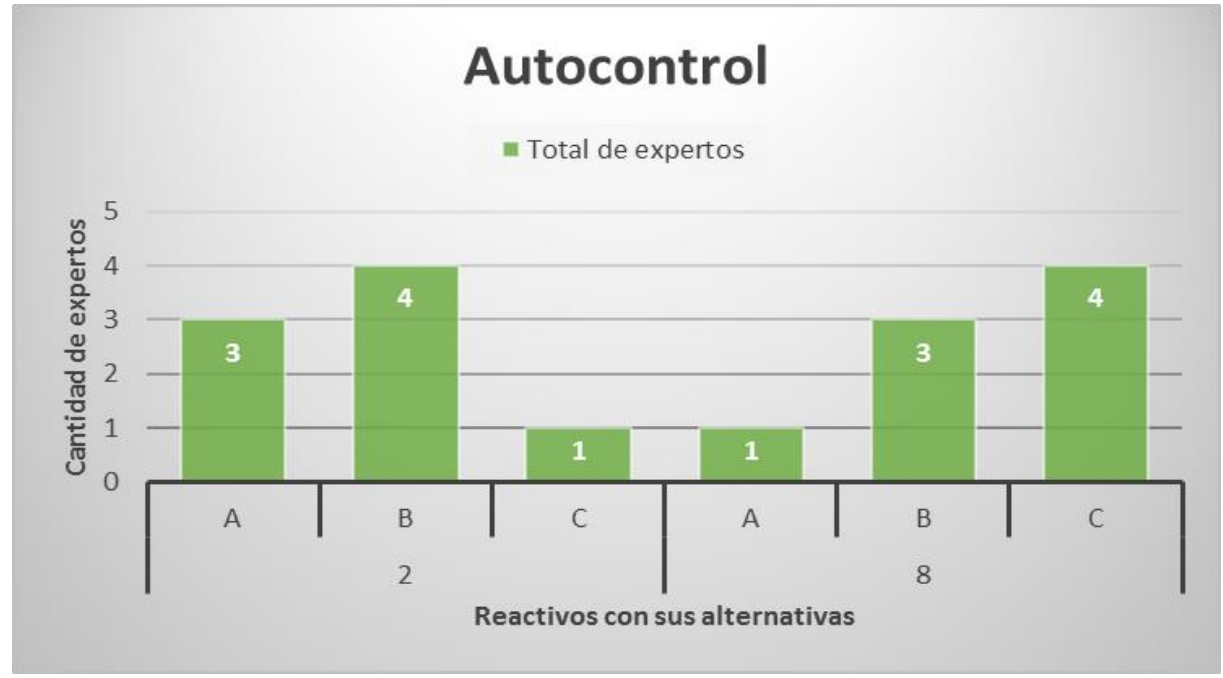

Fuente: Elaboración propia.

La figura 4 ilustra el análisis del diagnóstico emocional (empatía), donde el reactivo 3 la alternativa (a) podría ser adecuada en algunas circunstancias, la alternativa (b) indica sensibilidad ante un posible problema emocional por parte de la otra persona y la alterativa (c) demuestra la falta de sensibilidad por el sentimiento ajeno. Se puede observar que la mayoría de los expertos tienen sensibilidad por el sentimiento ajeno. En el reactivo 6 la alternativa (a) indica la habilidad para reconocer diferentes emociones, pero sin dejarse llevar por ellas. La alternativa (b) reconoce diferentes perspectivas emocionales; sin embargo, podría generar sentimientos negativos o de pérdidas emocionales. La alternativa (c) no reconoce los diferentes compromisos emocionales. Se distingue que la mayoría de los expertos reconocen las emociones de sus compañeros, pero no sensibilizan con las mismas. 
En el reactivo 9 la alternativa (c) podría ser adecuada en algunas circunstancias, aunque sólo la alternativa (b) reconoce de manera empática los sentimientos de la otra persona. Se puede notar que la mayoría de los expertos adoptaron la alternativa (b) como respuesta, por lo tanto, demuestran que son empáticos.

En conclusión, los reactivos 3, 6 y 9 dirigidos a la dimensión empatía refleja que los expertos tienen, aunque con algunas dificultades, la capacidad para comprender los sentimientos, emociones, preocupaciones, necesidades y puntos de vista de los otros, lo que permite una adecuada interacción con los otros, $\mathrm{y}$ un manejo factible de las problemáticas y emociones ajenas, además de un aprovechamiento y adaptación a la diversidad existente entre las personas.

Figura 4. Comportamiento del diagnóstico emocional (empatía).

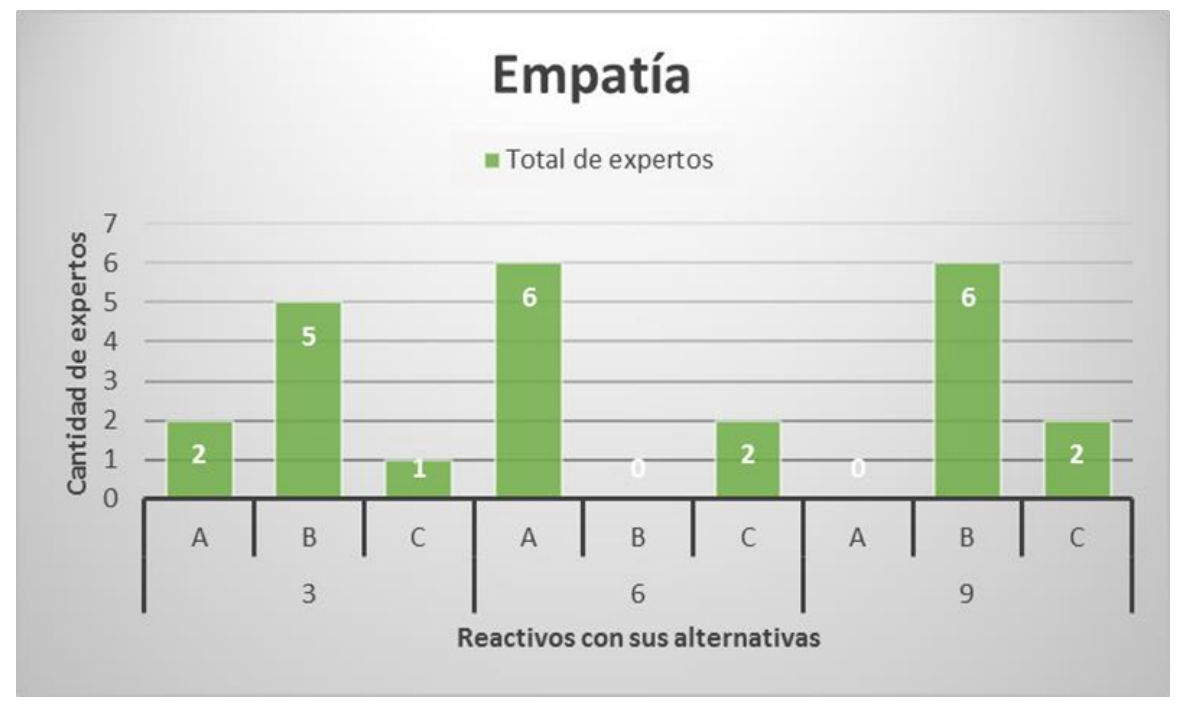

Fuente: Elaboración propia.

El comportamiento de la respuesta emocional (Manejo de relaciones) se muestra en la figura 5, donde el reactivo 4 las alternativas (a) y (c) podrían indicar que no es sensible al clima emocional del grupo, y su comportamiento podría ser inadecuado. La alternativa (b) demuestra la capacidad para establecer buena conexión con el grupo. Se aprecia que la mayoría de los expertos optaron por la alternativa (a) como respuesta, lo que demuestra, que no tienen la capacidad de trabajar en equipo. En el reactivo 10 la alternativa (a) indica permanecer bajo control, la alternativa (b) significa la capacidad de manejar los conflictos, aunque con algunas dificultades y la alternativa (c) implica la pérdida de control emocional en momento de confrontación. Se percibe que los expertos al elegir la alternativa (c) como respuesta, no son capaces de manejar los conflictos.

Se llega a la conclusión de que los reactivos 4 y 10 dedicados a la dimensión manejo de relaciones valora que los expertos no manejan adecuadamente las emociones en las 
relaciones interpersonales. Además, se les dificulta persuadir, influir, inspirar, dirigir, comunicar y negociar con los demás, ya sea una persona o un grupo.

Figura 5. Comportamiento de la respuesta emocional (Manejo de relaciones).

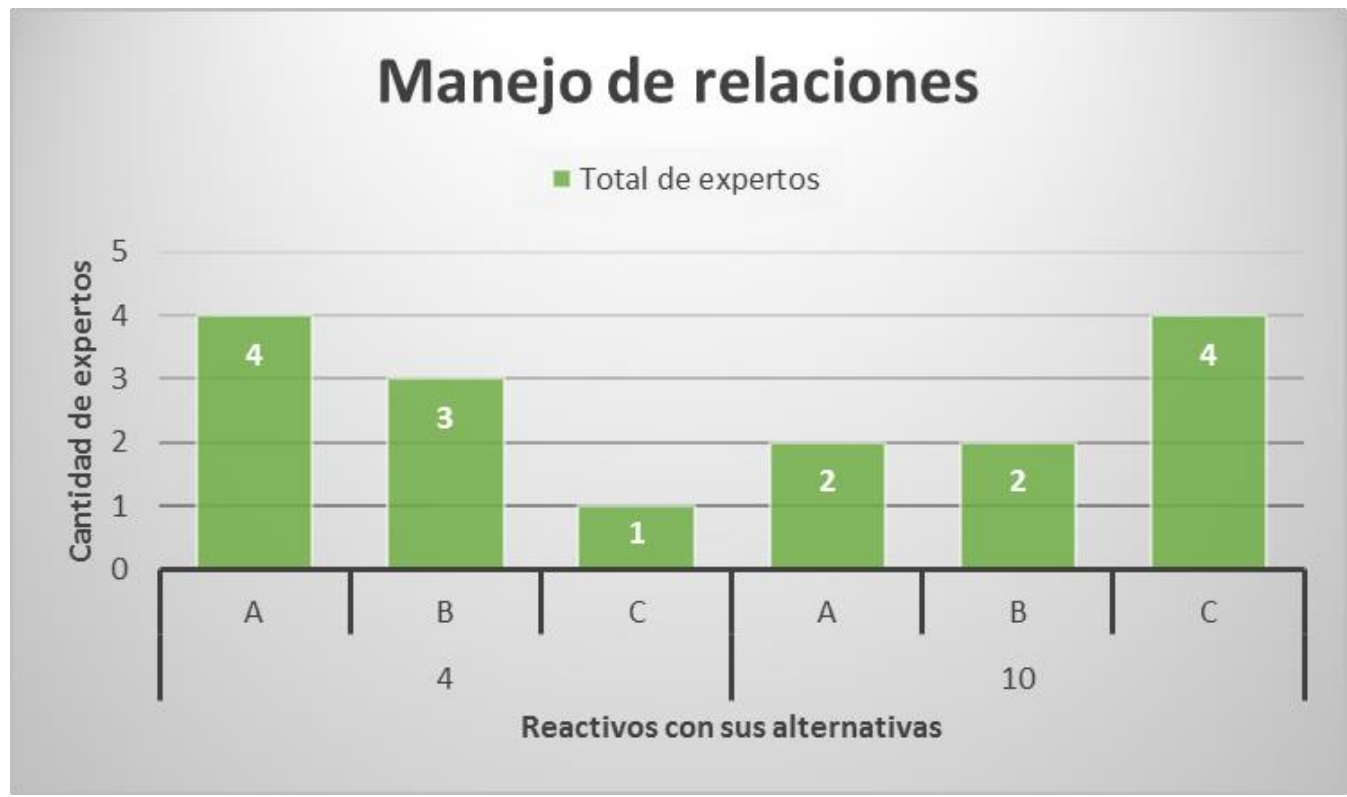

Fuente: Elaboración propia.

La entrevista que se realizó a la directora general del Hotel NH Capri perteneciente a la cadena hotelera Gran Caribe, se aprecia que el conocimiento de la inteligencia emocional no es muy amplio. Al mencionar las capacidades y características que un buen directivo debe tener se hace perceptible como pasan por alto otras de suma importancia para el desarrollo exitoso de la gestión en la entidad como lo es la inteligencia emocional. Otro resultado que arrojó la entrevista fue la insuficiente aplicación de habilidades directivas, destacando la falta de comunicación. En un caso específico se habla de la inteligencia emocional como una herramienta cotidiana, que una vez adquirida se vuelven casi imperceptibles para el directivo. Otro resultado de la entrevista es la no existencia en la instalación de programas de formación dirigidas al desarrollo e implementación de la inteligencia emocional, lo que dificulta a los directivos el conocimiento de esta temática. Como resultado final se aprecia que hay poco conocimiento y dominio de esta temática $\mathrm{y}$, en consecuencia, no son lo suficientemente empáticos, no manejan bien las relaciones con sus colegas, entre otras deficiencias.

\section{Propuesta de acciones para desarrollar la inteligencia emocional en los directivos del hotel NH Capri.}

1. Establecer requisitos de preparación del personal en los contratos de trabajo.

Explicación: Implica la evaluación del nivel de preparación de los trabajadores por medio de entrevistas y encuestas sistemáticas. 
2. Capacitar al personal en actuales tendencias directivas, operacionales y estratégicas.

Explicación: Impartir cursos de superación profesional.

3. Ofrecer cursos de Inteligencia emocional y su relación con la habilidad comunicativa.

Explicación: Consiste en impartir cursos que desarrollen la inteligencia emocional y que capaciten a los directivos en habilidades comunicativas con el objetivo de lograr una comunicación adecuada.

4. Intencional la escucha activa en las conversaciones con otros trabajadores.

Explicación: Se refiere a que en un horario determinado en el día los directivos se reúnan con sus subordinados y practiquen la escucha activa. La escucha activa se centra no solamente en las palabras, es decir, que tiene en cuenta el lenguaje no verbal (gestos, posturas, mímicas, etc.) y la comunicación de las emociones.

5. Realizar talleres sobre reconocimiento de emociones propias y ajenas.

Explicación: Los talleres consisten en realizar debates, lluvia de ideas, grupos de discusión y paneles donde se reconozcan las emociones. Se refiere a que en momento de emoción los directivos tengan la capacidad para detenerse un momento, aclarar la mente y responder y tomar decisiones con la cabeza más que con el corazón.

6. Implementar sesiones de coaching con los directivos.

Explicación: Consiste en que cada directivo tenga un encuentro con el coach al menos una vez por semana. Un coach es un experto en desarrollo personal que los ayudará a conocerse mejor y a desarrollar sus habilidades emocionales, además de acompañarlos en el proceso de cambio para que mejoren su bienestar y superen sus creencias limitantes.

7. Controlar los pensamientos y cómo te comportas.

Explicación: Se reseña que en los momentos emocionales se detengan e identifiquen la emoción para que no se pierda el control. Los sentimientos son el resultado de la emoción y de lo que se piensa sobre esa emoción. No se puedes evitar la emoción, pero sí modificar los pensamientos al respecto.

8. Analizar las críticas de la mejor manera.

Explicación: Consiste en aprender a aceptar las críticas, estar demasiado pendiente de lo que los demás dicen de si es un síntoma de que no se tienes autoconfianza y pueden hacer que se accedas a la ira.

9. Identificar tus fortalezas y debilidades. 
Explicación: Consiste en la identificación de las fortalezas y debilidades propias y elegir a qué extremo se quiere prestar atención. Se resume en utilizar las fortalezas y aprender de las debilidades.

10. Realizar talleres donde predomine los enfrentamientos a las emociones negativas.

Explicación: Se refiere a tener encuentros con los trabajadores donde se aborde los sentimientos negativos como la ira, la decepción, frustración, tristeza... de frente, de manera resolutiva y sin victimismo. Evadirlas para no pensar en ellas a la larga causará más problemas.

11. Aumentar la motivación del personal mediante el reconocimiento moral e incentivos salariales.

Explicación: Implica establecer criterios de evaluación para todos los directivos y reconocer moral y salarialmente a los más destacados.

12. Emitir las emociones de forma asertiva.

Explicación: En una situación de confrontación, el asertividad es la manera más eficaz de gestionar los conflictos. No lo confundas con pasividad o evitación. El asertividad es la capacidad para gestionar mejor las situaciones de confrontación. Aprende a decir "no" de una manera asertiva y establece tus prioridades.

13. Capacitar a los directivos para lograr la captación o percepción de mensajes no verbales o lenguaje extraverbal.

Explicación: Consiste en impartir cursos de lenguaje extraverbal. Se refiere a la capacidad con la que los directivos perciben o captan las señales no verbales que le trasmite otra persona, tales como gestos, posturas, mímicas, etc.

14. Capacitar al personal en técnicas de resolución de conflictos.

Explicación: Lo que se quiere lograr es que se obtenga la capacidad que le permite al directivo negociar y resolver conflictos haciendo uso de determinadas estrategias y procedimientos personales.

15. Implantar el trabajo en equipo para la toma de decisiones.

Explicación: Se refiere a la competencia que merita el trabajo en equipo, como ser capaces de crear unión grupal en la consecución de las metas colectivas y establecer unas adecuadas relaciones interpersonales.

16. Establecer un ambiente de adecuada relación grupal a partir de actividades recreativas. 
Explicación: Se refiere a la programación de actividades culturales y deportivas que contribuyan al desarrollo de la inteligencia emocional y consoliden las relaciones interpersonales para así crear un ambiente ameno.

17. Programar actividades que contribuyan al desarrollo de la inteligencia emocional.

Explicación: Consiste en realizar una vez al mes en horario no laborable festivales culturales de las distintas manifestaciones artísticas, festivales y competencias deportivas dentro de la instalación.

18. Aplicar mensualmente técnicas de evaluación de la inteligencia emocional para conocer el nivel que poseen los directivos.

Explicación: Se refiere a la aplicación de entrevistas, encuestas y test de inteligencia emocional.

\section{Conclusiones.}

Luego de desarrollar la presente investigación se arriba a las siguientes conclusiones:

- Se evidenció que, los directivos evaluados presentan un nivel medio-bajo de inteligencia emocional, por lo que se hace necesario el desarrollo e implementación de dicha habilidad para poder alcanzar buen desempeño en el puesto de trabajo.

- La propuesta de acciones para desarrollar la inteligencia emocional garantiza que los directivos amplíen su capacidad de influencia y liderazgo, manejen mejor las relaciones interpersonales, favorezcan su bienestar psicológico y mejoren su rendimiento laboral motivándose cada vez más en desempeñar eficiente y eficazmente su rol como directivo. Es necesario explotar al máximo aquellas capacidades que nos permitan tener unas mejores relaciones con las personas que se encuentran a nuestro alrededor, así como con nosotros mismos. Desarrollando dichas habilidades, también estaremos en condición de ser verdaderos líderes, ya que el trato que le daremos a nuestros posibles subordinados, será de total cordialidad, exigiendo mucho pero no irrespetando.

\section{Referencias bibliográficas.}

Bello, Z. (2012). Ser inteligente es mucho más. La Habana: Felix Varela.

Calzado, R. E. (2020). Acciones para el desarrollo de la inteligencia emocional como habilidad directiva en los cuadros del Hotel NH Capri. Tesis de Diploma en opción al título de Licenciado en Turismo. Facultad de Turismo. Universidad de la Habana. La Habana.73p.

Cooper, K. (1998). "La inteligencia emocional, aplicada al liderazgo y a las organizaciones". Bogotá: Norma S.A. 
Goleman, D. (1995). Inteligencia Emocional. Buenos Aires: Javier Vergara Editor S.A.

Goleman, D. (1998). La práctica de la inteligencia emocional. Barcelona, España: Kairós S.A.

Hernández, R. F. C. (2010). Metodología de la investigación. México D.F.: Quinta edición MxGrawHill Educacion.

Martínez, C. M. (2017). Procedimiento para la capacitación en negociación a directivos hoteleros.

Mayer, S. (1990). Emotional intelligence. Imagination, cognition and personality.

Ronda. (2007). Dirección Estratégica. Construcción y Dimensiones. La Habana, Cuba: Ediciones Futuro. 
Para citar el artículo indexado

Calzado Morales, R. E., Valdés Bencomo, Y., \& Vázquez Alfonso, Y. (2020). Acciones para el desarrollo de la inteligencia emocional en los cuadros del Hotel NH Capri. AlfaPublicaciones, 2(2), 79-95. https://doi.org/10.33262/ap.v2i2.30

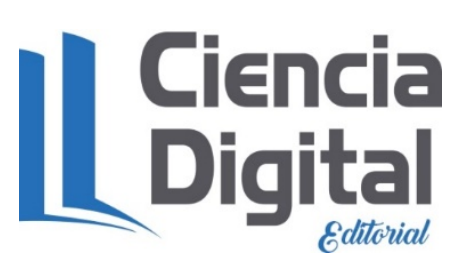

El artículo que se publica es de exclusiva responsabilidad de los autores y no necesariamente reflejan el pensamiento de la Revista Alpha Publicaciones.

El artículo queda en propiedad de la revista y, por tanto, su publicación parcial y/o total en otro medio tiene que ser autorizado por el director de la Revista Alpha Publicaciones.
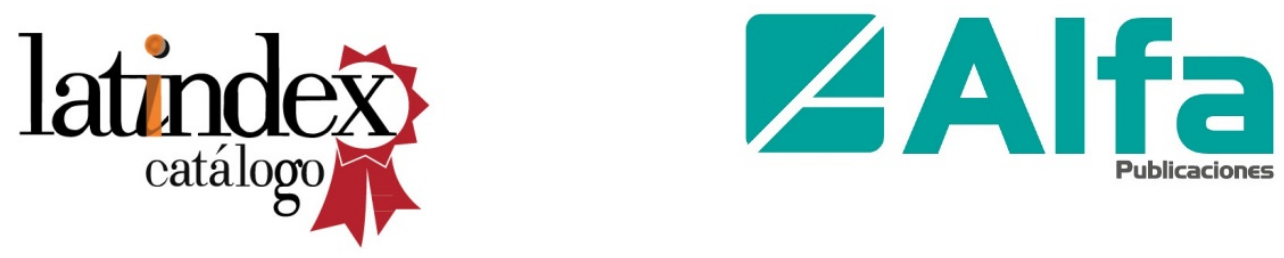